\title{
Evaluation of Hospital Information Systems in Iran: A Case Study in the Kerman Province
}

\author{
Somayeh Noori Hekmat ${ }^{1}$, Reza Dehnavieh ${ }^{2}$, Tahereh Behmard ${ }^{3}$, Razieh Khajehkazemi ${ }^{4}$, \\ Mohammad Hossain Mehrolhassani ${ }^{2} \&$ Atousa Poursheikhali ${ }^{5}$ \\ ${ }^{1}$ Health Services Management Research Center, Institute for Futures Studies in Health, Kerman University of \\ Medical Sciences, Kerman, Iran \\ ${ }^{2}$ Medical Informatics Research Center, Institute for Futures Studies in Health, Kerman University of Medical \\ Sciences, Kerman, Iran \\ ${ }^{3}$ Kerman University of Medical Science, Kerman, Iran \\ ${ }^{4}$ Modeling in Health Reseach Center, Institute for Futures Studies in Health, Kerman University of Medical \\ Sciences, Kerman, Iran \\ ${ }^{5}$ Social Determinants of Health Research Center, Institute for Futures Studies in Health, Kerman University of \\ Medical Sciences, Kerman, Iran \\ Correspondence: Atousa Poursheikhali, Social Determinants of Health Research Center, Institute for Futures \\ Studies in Health, Kerman University of Medical Sciences, Kerman, Iran. Tel: 98-34-3132-5402. E-mail: \\ Atousa_prsh@yahoo.com
}

Received: January 21, 2016

doi:10.5539/gjhs.v8n12p95
Accepted: February 26, 2016 Online Published: April 29, 2016

URL: http://dx.doi.org/10.5539/gjhs.v8n12p95

\begin{abstract}
The Hospital Information system (HIS) is a comprehensive solution that offers complete data integration for different administrative levels in hospitals. To the extent that this system is close to its aim, the efficiency and quality of health care would increase in hospitals. The performance of HIS systems in 13 hospitals in Kerman province that they were evaluated based on four major criteria of ownership, location, education and software design. Seven hospitals were located in the capital city of Kerman province. According to teaching status of hospitals, four were teaching and based on their ownership three were public. The checklist of Iranian ministry of health and medical education, containing 20 indexes were used to evaluate each hospital's HIS system in three main supportive, diagnosis and clinical sectors. Spearman correlation coefficient was used to assess the association between major sectors. The highest score $(\operatorname{mean} \pm \mathrm{SD})$ was observed in laboratory information systems (88.19 \pm 13.69$)$, resource management ( $84.47 \pm 8.94)$, and registration information systems $(84.47 \pm 18.06)$; the lowest scores were for telemedicine (45.58 \pm 3.86$)$, staff information and timing systems $(40 \pm 16.64)$, and decision support systems (23.6 4 4.97). The total score of HIS software was positively correlated with all its three components. There were strong positive correlations between all three components. The three factors of decision support systems, staff information systems and telemedicine have an important role in providing solutions for non-structured management problems and for leading decision-makers to insights, improving human resource management and solving the problem of access to services. Thus, based on the survey findings, those three factors need to be improved in the Iranian hospital information system.
\end{abstract}

Keywords: hospital information systems, decision support system, staff information system, telemedicine, resource management system, laboratory information systems

\section{Introduction}

As massive global changes modify the rules, information has an impressive role in effective decision making, which has led to a vast investment in information technologies in healthcare organizations with the aim of increasing the quality of services and reducing healthcare costs (Nurek, Kostopoulou, Delaney, \& Esmail, 2015; Sadeghifar, Jafari, Tofighi, Ravaghi, \& Maleki, 2015). Due to the importance of the issue, health information systems were introduced in 1970 (Hannan, 2014). The experiences of developed countries in the health care system demonstrated that optimal preparation and utilization of information technology has a vital role in the production and quality of health services (Schoen et al., 2012). Therefore, systematic evaluation of hospital 
information systems can support the clinical, diagnostic and supportive proceedings, and led to the software's enhancement and development based on users' needs (Chatzoglou, Fragidis, Doumpa, \& Aggelidis, 2012).

Implementation of health information systems results in an increase of the quality of patient care and safety through reducing medication errors, improving providers' performance and effectiveness of service, reducing treatment costs, and conserving resources in health and medical organizations (Dehnavieh et al., 2014). Moreover, these systems can increase the legibility of recorded data, reduce medical errors and lead to users' satisfaction (Ahmadian, Nejad, \& Khajouei 2015; Jouyani et al., 2013; Vafaee-Najar et al., 2013).

The main purpose of the development of health information systems is to integrate health information to meet the needs of patients, epidemiological research, quality of care enhancement, reduce costs, and automate tasks such as reporting test results, entering the physician's order, administration, central warehouse, feeding unit, etc. Hospital information systems (HIS) cover health information systems in the treatment aspect by following all main purposes in the hospital field (Dolan, 2015), which has an important role in the healthcare sector as an industry, and which needs to be improved continuously, especially in the context of healthcare management (Ismail, Abdullah, \& Shamsuddin, 2015). The implementation of health information systems has an increasingly important place in healthcare sector because of the significance of its role, and they need to manage and integrate clinical, financial and operational information (Thakare \& Khire, 2014). Hospital information systems have been designed to support healthcare services and information as an integral member of management, such as the computer systems most frequently used in healthcare (Ajami \& Mohammadi-Bertiani, 2013).

Hospital information systems need to be customized in a way that fits the context. In that regard, the role of software vendors is an important one, in that they guarantee the fitness of the software based on specific requirements from hospitals that are their direct users (Balaraman \& Kosalram, 2013), and the HIS can enhance patient access to better healthcare costs and improve quality of health care delivery (Jahanbakhsh, Sharifi, \& Ayat, 2014).

In Iran, there is at least one medical science university in each province, which acts as an official representative of the Ministry Of Health and Medical Education (MOHME) in that province (Hekmat et al., 2015; Hekmat et al., 2014; Turani et al., 2011). Hospitals can be divided into categories based on different criteria. For example, we can classify hospitals to private and public categories based on the hospitals' ownership, or whether public hospitals are teaching or non- teaching (Sirizi et al., 2008). There is not enough information about HIS performance in these hospitals, so it is necessary to design a study for gathering information about the situation of HIS domains. The purpose of this survey is to evaluate the different aspects of HIS in Kerman province hospitals by investigating all types of hospitals to compare their functionality in HIS indexes.

\section{Method}

This descriptive analytical study was conducted among all hospitals located in Kerman province which were equipped with HIS (except social security and military hospitals) in 2013. We assessed 13 hospitals; seven were located in Kerman city, the capital city of Kerman province. According to teaching status of hospitals, four were teaching (nine non-teaching) and based on their ownership, three were private (10 public).

A standard checklist provided by MOHME was used to evaluate each hospital's HIS software. Its validity and reliability have been approved by MOHME. It was comprised of 20 indexes. Each index was belonged to one of the three components of clinical, diagnostic and supportive.

In a joint meeting with hospital's information system administrators, they were asked to complete the checklist based on the current HIS software in their hospital. For each index, three states were designed by MOHME, as follows: 1) this function is completely defined in our HIS software, with all its required sub functions and we use it; 2) this function is designed in our HIS software, but we don't use it; 3) this function has not been designed in our HIS software, we need to modify the software to include it. The scores of $1,0.5$, and zero were used for the three choices, respectively. The evaluation score for each index and the total score across all indexes were calculated out of 100 .

Descriptive statistics including mean and standard deviation (SD) were calculated to report indexes in different hospitals by their teaching status, ownership, geographical location, and type of companies. To assess the relationship between three components and the overall score, the Spearman correlation coefficient was used. The Mann-Whitney $U$ test was used to compare the 20 indexes, three components and the overall score by their teaching status (teaching vs. non-teaching), ownership (private vs. public), and geographical location (located in the capital city of Kerman vs. other cities within Kerman province). The Kruskal-Wallis test was used to compare the 20 indexes, three components and the overall score by their software designed companies. All 
analysis was done using SPSS (version 16). A p-value less than 0.05 were considered as a significant.

\section{Results}

The highest mean evaluation scores were found for laboratory information systems (88.19), resource management (84.47), and registration information systems (84.47); while the lowest were observed for telemedicine (45.58), staff information and timing systems (40), and decision support systems (23.6) (Table 1). In public hospitals the highest mean evaluation scores were observed for laboratory information system (87.87), resource management (85.58), and registration information systems (82.22); while for private hospitals, the security services (94.49), discharge information systems (92.17), and patient admission systems (91.96) had the highest scores. However, the lowest mean evaluation scores were nursing electronic records (40.15), electronic medical records (40.16), and decision support systems (11.80) in public hospitals and decision support systems (63.06), personnel staffing and scheduling information systems (52.22), and special records (45.71) in private hospitals. The comparison of 20 indexes between public and private hospitals showed that private hospitals had a significant greater mean score for electronic medical records, decision support systems, security services, and telemedicine service indexes (Table 1).

In hospitals located in the capital city of Kerman province, the highest mean evaluation scores were observed for laboratory information systems (91.97), discharge information systems (89.37), and resource management places (89.27); while in other cities laboratory information systems (83.79), patient admission systems (81.57), and bed management places (81.65) had the highest scores. However, the lowest mean evaluation scores were special records (55.48), telemedicine systems place (53.17), and decision support systems (37.56) in hospital located in the capital city and electronic medical records (31.44), staff informational systems (18.88), and decision support systems (7.35) in other cities. The comparison of 20 indexes by geographical location of hospitals did not show any significant difference (Table 1).In teaching hospitals the first three highest mean evaluation scores were obtained for patient admission systems (95.2), laboratory information systems (93.99), and warehouse management (92.27); while for non-teaching hospitals, laboratory information systems (85.61), patient admission systems (85.3), and discharge information systems (82.93) had the highest scores. However, the three lowest score were nursing electronic records (50.58), telemedicine services (41.37), and decision support systems (18.46) in teaching hospitals and special records (43.94), staff informational systems (30), and decision support systems (25.90) in non-teaching hospitals. The comparison of 20 indexes by teaching status of hospitals showed that teaching hospitals had a significant mean score for medical electronic records compared to non-teaching hospitals ( $\mathrm{p}$-value $=0.04$ ).

Table 1. Distribution of evaluation of 20 indexes by hospital ownership and teaching statuses and its geographical location

\begin{tabular}{|c|c|c|c|c|c|c|c|c|c|c|c|}
\hline \multirow{2}{*}{ Indexes } & & \multirow{2}{*}{$\begin{array}{l}\text { Mean } \\
(\mathrm{SD})\end{array}$} & \multicolumn{3}{|c|}{$\begin{array}{l}\text { Hospital ownership status } \\
\text { Mean (SD) }\end{array}$} & \multicolumn{3}{|c|}{$\begin{array}{l}\text { Geographical location } \\
\text { Mean (SD) }\end{array}$} & \multicolumn{3}{|c|}{$\begin{array}{l}\text { Hospital teaching status } \\
\text { Mean (SD) }\end{array}$} \\
\hline & & & Public & Private & P-value & $\begin{array}{l}\text { Capital } \\
\text { city }\end{array}$ & $\begin{array}{l}\text { Other } \\
\text { cities }\end{array}$ & P-value & Teaching & Non-teaching & P-value \\
\hline \multirow{6}{*}{ Clinical } & $\begin{array}{l}\text { Medical } \\
\text { electronic } \\
\text { records }\end{array}$ & $\begin{array}{l}49.39 \\
(54.34)\end{array}$ & $\begin{array}{l}40.16 \\
(25.76)\end{array}$ & $\begin{array}{l}80.15 \\
(0)\end{array}$ & $0.01 *$ & $\begin{array}{l}64.75 \\
(18.04)\end{array}$ & $\begin{array}{l}31.44 \\
(27.97)\end{array}$ & 0.31 & $\begin{array}{l}53.84 \\
(16.00)\end{array}$ & $47.41(33.12)$ & $0.04 *$ \\
\hline & Nursing records & $\begin{array}{l}50.30 \\
(22.76)\end{array}$ & $\begin{array}{l}40.15 \\
(32.226)\end{array}$ & $\begin{array}{l}84.12 \\
(0)\end{array}$ & 0.12 & $\begin{array}{l}64.95 \\
(27.33)\end{array}$ & $\begin{array}{l}33.19 \\
(39.19)\end{array}$ & 0.31 & $\begin{array}{l}50.58 \\
(29.19)\end{array}$ & $50.17(40.46)$ & 0.11 \\
\hline & $\begin{array}{l}\text { Bed } \\
\text { management }\end{array}$ & $\begin{array}{l}80.34 \\
(6.48)\end{array}$ & $\begin{array}{l}81.81 \\
(13.23)\end{array}$ & $\begin{array}{l}75.45 \\
(0)\end{array}$ & 0.43 & $\begin{array}{l}79.21 \\
(12.78)\end{array}$ & $\begin{array}{l}81.65 \\
(11.54)\end{array}$ & 0.31 & $\begin{array}{l}82.03 \\
(17.38)\end{array}$ & $79.58(9.63)$ & 0.71 \\
\hline & Special records & $\begin{array}{l}49.77 \\
(7.83)\end{array}$ & $\begin{array}{l}51.00 \\
(25.68)\end{array}$ & $\begin{array}{l}45.71 \\
(0)\end{array}$ & 0.99 & $\begin{array}{l}55.48 \\
(20.31)\end{array}$ & $\begin{array}{l}43.08 \\
(24.60)\end{array}$ & 0.31 & $\begin{array}{l}62.85 \\
(25.65)\end{array}$ & $43.94(19.48)$ & 0.14 \\
\hline & $\begin{array}{l}\text { Personnel } \\
\text { Staffing And } \\
\text { Scheduling } \\
\text { Information } \\
\text { Systems }\end{array}$ & $\begin{array}{l}40.00 \\
(16.64)\end{array}$ & $\begin{array}{l}36.33 \\
(41.93)\end{array}$ & $\begin{array}{l}52.22 \\
(0)\end{array}$ & 0.29 & $\begin{array}{l}58.08 \\
(34.28)\end{array}$ & $\begin{array}{l}18.88 \\
(29.66)\end{array}$ & 0.31 & $\begin{array}{l}62.48 \\
(47.86)\end{array}$ & $30.00(28.75)$ & 0.05 \\
\hline & $\begin{array}{l}\text { Decision } \\
\text { support systems }\end{array}$ & $\begin{array}{l}23.62 \\
(16.97)\end{array}$ & $\begin{array}{l}11.80 \\
(23.92)\end{array}$ & $\begin{array}{l}63.06 \\
(0)\end{array}$ & $0.02 *$ & $\begin{array}{l}37.56 \\
(35.35)\end{array}$ & $\begin{array}{l}7.35 \\
(24.05)\end{array}$ & 0.99 & $\begin{array}{l}36.93 \\
(18.46)\end{array}$ & $25.90(29.51)$ & 0.15 \\
\hline
\end{tabular}




\begin{tabular}{|c|c|c|c|c|c|c|c|c|c|c|c|}
\hline & $\begin{array}{l}\text { Services } \\
\text { Terminology }\end{array}$ & $\begin{array}{l}65.02 \\
(14.69)\end{array}$ & $\begin{array}{l}61.27 \\
(33.19)\end{array}$ & $\begin{array}{l}64.70 \\
(0)\end{array}$ & 0.43 & $\begin{array}{l}68.90 \\
(28.23)\end{array}$ & $\begin{array}{l}54.07 \\
(29.86)\end{array}$ & 0.31 & $\begin{array}{l}72.05 \\
(13.50)\end{array}$ & $57.60(24.19)$ & 0.61 \\
\hline & $\begin{array}{l}\text { Security } \\
\text { Services }\end{array}$ & $\begin{array}{l}68.69 \\
(31.54)\end{array}$ & $\begin{array}{l}60.96 \\
(28.77)\end{array}$ & $\begin{array}{l}94.49 \\
(0)\end{array}$ & $0.04 *$ & $\begin{array}{l}80.20 \\
(20.81)\end{array}$ & $\begin{array}{l}55.27 \\
(33.66)\end{array}$ & 0.31 & $\begin{array}{l}69.49 \\
(22.58)\end{array}$ & $68.34(36.62)$ & 0.06 \\
\hline & $\begin{array}{l}\text { Communication } \\
\text { services }\end{array}$ & $\begin{array}{l}68.64 \\
(12.06)\end{array}$ & $\begin{array}{l}66.19 \\
(30.43)\end{array}$ & $\begin{array}{l}72.52 \\
(0)\end{array}$ & 0.99 & $\begin{array}{l}75.49 \\
(11.05)\end{array}$ & $\begin{array}{l}58.50 \\
(36.79)\end{array}$ & 0.31 & $\begin{array}{l}77.73 \\
(15.14)\end{array}$ & $63.18(29.91)$ & 0.75 \\
\hline & $\begin{array}{l}\text { Telemedicine } \\
\text { services }\end{array}$ & $\begin{array}{l}45.58 \\
(3.84)\end{array}$ & $\begin{array}{l}38.62 \\
(26.62)\end{array}$ & $\begin{array}{l}68.96 \\
(0)\end{array}$ & $<0.01^{*}$ & $\begin{array}{l}53.17 \\
(24.41)\end{array}$ & $\begin{array}{l}36.75 \\
(28.48)\end{array}$ & 0.99 & $\begin{array}{l}41.37 \\
(27.58)\end{array}$ & $47.44(27.65)$ & 0.11 \\
\hline & $\begin{array}{l}\text { Outpatient } \\
\text { information } \\
\text { systems }\end{array}$ & $\begin{array}{l}78.65 \\
(9.63)\end{array}$ & $\begin{array}{l}78.65 \\
(9.63)\end{array}$ & $\begin{array}{l}88.63 \\
(0)\end{array}$ & 0.60 & $\begin{array}{l}77.90 \\
(26.54)\end{array}$ & $\begin{array}{l}79.54 \\
(17.63)\end{array}$ & 0.31 & $\begin{array}{l}69.88 \\
(34.77)\end{array}$ & $82.56(14.45)$ & 0.88 \\
\hline & $\begin{array}{l}\text { Hospital Ward } \\
\text { Information } \\
\text { Systems }\end{array}$ & $\begin{array}{l}75.76 \\
(8.31)\end{array}$ & $\begin{array}{l}75.76 \\
(8.31)\end{array}$ & $\begin{array}{l}83.33 \\
(0)\end{array}$ & 0.30 & $\begin{array}{l}76.60 \\
(25.20)\end{array}$ & $\begin{array}{l}51.93 \\
(25.60)\end{array}$ & 0.31 & $\begin{array}{l}73.33 \\
(34.83)\end{array}$ & $62.4(25.6)$ & 0.08 \\
\hline & $\begin{array}{l}\text { Pharmacy } \\
\text { information } \\
\text { systems }\end{array}$ & $\begin{array}{l}71.37 \\
(9.36)\end{array}$ & $\begin{array}{l}71.37 \\
(9.36)\end{array}$ & $\begin{array}{l}79.32 \\
(0)\end{array}$ & 0.12 & $\begin{array}{l}74.93 \\
(47.64)\end{array}$ & $\begin{array}{l}62.21 \\
(9.74)\end{array}$ & 0.31 & $\begin{array}{l}71.64 \\
(13.48)\end{array}$ & $71.25(9.78)$ & 0.38 \\
\hline & $\begin{array}{l}\text { Operating room } \\
\text { information } \\
\text { systems }\end{array}$ & $\begin{array}{l}63.08 \\
(18.56)\end{array}$ & $\begin{array}{l}58.11 \\
(29.07)\end{array}$ & $\begin{array}{l}79.71 \\
(0)\end{array}$ & 0.30 & $\begin{array}{l}69.14 \\
(32.55)\end{array}$ & $\begin{array}{l}56.02 \\
(18.82)\end{array}$ & 0.31 & $\begin{array}{l}61.23 \\
(43.85)\end{array}$ & $63.92(19.01)$ & 0.71 \\
\hline \multirow{2}{*}{ Diagnostic } & $\begin{array}{l}\text { Laboratory } \\
\text { information } \\
\text { systems }\end{array}$ & $\begin{array}{l}88.19 \\
(13.69)\end{array}$ & $\begin{array}{l}87.87 \\
(12.52)\end{array}$ & $\begin{array}{l}89.28 \\
(0)\end{array}$ & 0.60 & $\begin{array}{l}91.97 \\
(5.19)\end{array}$ & $\begin{array}{l}82.79 \\
(14.40)\end{array}$ & 0.31 & $\begin{array}{l}93.99 \\
(6.42)\end{array}$ & $85.61(11.71)$ & 0.34 \\
\hline & $\begin{array}{l}\text { Radiology } \\
\text { information } \\
\text { systems }\end{array}$ & $\begin{array}{l}68.26 \\
(12.56)\end{array}$ & $\begin{array}{l}63.57 \\
(23.76)\end{array}$ & $\begin{array}{l}83.92 \\
(0)\end{array}$ & 0.12 & $\begin{array}{l}71.92 \\
(25.12)\end{array}$ & $\begin{array}{l}63.98 \\
(20.23)\end{array}$ & 0.31 & $\begin{array}{l}62.94 \\
(31.80)\end{array}$ & $70.62(18.83)$ & 0.17 \\
\hline \multirow{4}{*}{ Supportive } & $\begin{array}{l}\text { Resource } \\
\text { Management } \\
\text { Information } \\
\text { Systems }\end{array}$ & $\begin{array}{l}85.51 \\
(8.94)\end{array}$ & $\begin{array}{l}85.58 \\
(15.17)\end{array}$ & $\begin{array}{l}85.29 \\
(0)\end{array}$ & 0.79 & $\begin{array}{l}89.97 \\
(7.33)\end{array}$ & $\begin{array}{l}81.11 \\
(17.52)\end{array}$ & 0.31 & $\begin{array}{l}92.27 \\
(8.92)\end{array}$ & $82.51(14.01)$ & 0.18 \\
\hline & $\begin{array}{l}\text { Patient } \\
\text { admission } \\
\text { systems }\end{array}$ & $\begin{array}{l}84.47 \\
(18.06)\end{array}$ & $\begin{array}{l}82.22 \\
(14.05)\end{array}$ & $\begin{array}{l}91.96 \\
(0)\end{array}$ & 0.12 & $\begin{array}{l}86.96 \\
(7.47)\end{array}$ & $\begin{array}{l}81.57 \\
(23.82)\end{array}$ & 0.31 & $\begin{array}{l}95.20 \\
(11.11)\end{array}$ & 85.03 (14.92) & 0.88 \\
\hline & $\begin{array}{l}\text { Medical } \\
\text { records } \\
\text { information } \\
\text { systems }\end{array}$ & $\begin{array}{l}81.06 \\
(3.63)\end{array}$ & $\begin{array}{l}81.78 \\
(17.78)\end{array}$ & $\begin{array}{l}78.72 \\
(0)\end{array}$ & 0.11 & $\begin{array}{l}85.53 \\
(8.12)\end{array}$ & $\begin{array}{l}75.87 \\
(20.85)\end{array}$ & 0.31 & $\begin{array}{l}90.63 \\
(7.10)\end{array}$ & $76.80(63.23)$ & 0.10 \\
\hline & $\begin{array}{l}\text { Discharge } \\
\text { information } \\
\text { systems }\end{array}$ & $\begin{array}{l}84.01 \\
(13.12)\end{array}$ & $\begin{array}{l}81.19 \\
(12.11)\end{array}$ & $\begin{array}{l}92.17 \\
(0)\end{array}$ & 0.11 & $\begin{array}{l}89.37 \\
(6.00)\end{array}$ & $\begin{array}{l}78.33 \\
(13 . .80)\end{array}$ & 0.31 & $\begin{array}{l}87.27 \\
(8.52)\end{array}$ & 82.93 (12.92) & 0.14 \\
\hline
\end{tabular}

* P-value $<0.05$; SD: Standard deviation

Table 2. Distribution of evaluation of 20 indexes by software design companies

\begin{tabular}{llllll}
\hline \multirow{2}{*}{ Index } & \multicolumn{3}{l}{$\begin{array}{l}\text { Type of companies } \\
\text { Mean (SD) }\end{array}$} \\
\cline { 3 - 6 } & Tirazheh & $\begin{array}{l}\text { Peyvand } \\
\text { dadeh }\end{array}$ & Rahavard & $\begin{array}{l}\text { Noskheh } \\
\text { pardaz }\end{array}$ \\
\hline 1 & Resource Management Information Systems & $90.11(9.35)$ & $85.29(20.76)$ & $54.41(0)$ & $82.29(0)$ \\
\hline 2 & Medical electronic records & $\begin{array}{l}29.98 \\
(24.30)\end{array}$ & $65.27(1.93)$ & $61.22(0)$ & $80.15(0)$ \\
\hline 3 & Nursing records & $\begin{array}{l}21.07 \\
(21.22)\end{array}$ & $68.11(0.55)$ & $81.74(0)$ & $84.12(0)$ \\
4 & Bed management & $79.72(13.9)$ & $87.72(17.34)$ & $84.54(0)$ & $75.42(0)$ \\
\hline 5 & Special records & 43.65 & $59.28(1.0)$ & $85.71(0)$ & $45.71(0)$ \\
\hline
\end{tabular}




\begin{tabular}{|c|c|c|c|c|c|}
\hline & & $(26.51)$ & & & \\
\hline 6 & $\begin{array}{l}\text { Personnel Staffing And } \quad \text { Scheduling } \\
\text { Information Systems }\end{array}$ & $28.55(4.87)$ & $57.22(10.2)$ & $48.77(0)$ & $52.22(0)$ \\
\hline 7 & Decision support systems & $\begin{array}{l}10.45 \\
(27.90)\end{array}$ & $7.20(10.18)$ & $29.72(0)$ & $63.06(0)$ \\
\hline 8 & Terminology services & $\begin{array}{l}50.98 \\
(33.60)\end{array}$ & $82.35(24.94)$ & $91.17(0)$ & $34.96(0)$ \\
\hline 9 & Security services & $\begin{array}{l}47.96 \\
(23.77)\end{array}$ & $90.36(11.02)$ & $93.11(0)$ & $94.49(0)$ \\
\hline 10 & Communication services & $\begin{array}{l}54.76 \\
(29.33)\end{array}$ & $91.20(12.41)$ & $96.15(0)$ & $64.70(0)$ \\
\hline 11 & Telemedicine services & $\begin{array}{l}31.51 \\
(29.44)\end{array}$ & $5.71(0)$ & $55.17(0)$ & $68.96(0)$ \\
\hline 12 & Patient admission systems & $\begin{array}{l}77.14 \\
(14.00)\end{array}$ & $93.39(2.77)$ & $97.17(0)$ & $91.96(0)$ \\
\hline 13 & Outpatient information systems & $\begin{array}{l}65.25 \\
(21.70)\end{array}$ & $100(0)$ & $100(0)$ & $88.63(0)$ \\
\hline 14 & Hospital Ward Information Systems & $50.46(26.2)$ & $100(0)$ & $51.66(0)$ & $71.42(0)$ \\
\hline 15 & Pharmacy information systems & $\begin{array}{l}64.96 \\
(10.27)\end{array}$ & $80.72(4.33)$ & $73.74(0)$ & $79.32(0)$ \\
\hline 16 & laboratory information systems & $\begin{array}{l}85.28 \\
(12.99)\end{array}$ & $99.80(8.88)$ & $82.14(0)$ & $82.28(0)$ \\
\hline 17 & Radiology information systems & $\begin{array}{l}57.14 \\
(25.41)\end{array}$ & $83.03(11.35)$ & $69.640)$ & $83.95(0)$ \\
\hline 18 & Operating room information systems & $\begin{array}{l}47.82 \\
(26.94)\end{array}$ & $92.75(10.24)$ & $60.86(0)$ & $79.71(0)$ \\
\hline 19 & Medical records information systems & $\begin{array}{l}78.25 \\
(20.04)\end{array}$ & $92.55(10.51)$ & $85.10(0)$ & $78.72(0)$ \\
\hline 20 & Discharge information systems & $\begin{array}{l}77.51 \\
(11.15)\end{array}$ & $88.26(5.53)$ & $100(0)$ & $92.17(0)$ \\
\hline
\end{tabular}

SD: Standard deviation

The results showed that HIS software was designed by four different companies. Table 2 shows our findings on evaluation of indexes by software design companies.

The Spearman's correlation coefficient showed the total evaluation score of HIS software is positively correlated with all three components. Moreover, there were strong positive correlations between all three components of the HIS software (Table 3). It means that an increase in evaluation score of each component is associated with an increase in the other components evaluation score and total evaluation score.

We have also found that there were not any significant correlations between the HIS software evaluation score (including the three components and overall) and the number of hospital's beds.

Table 3. The Spearman correlation coefficients between three components and total evaluation of HIS software

\begin{tabular}{lllll}
\hline & Supportive score & Diagnostic score & Clinical score & Overall score \\
\hline Supportive score & 1 & & & \\
Diagnostic score & 0.73 & 1 & & \\
Clinical score & 0.93 & 0.88 & 1 & 1 \\
Overall score & 0.8 & 0.98 & 0.92 & \\
\hline
\end{tabular}

Comparing different components of HIS software showed that respondents in private hospitals and respondents in teaching hospitals had better evaluation on all of the software components, but these differences were not statistically significant. Respondents in the hospitals which were located in Kerman city had better evaluation on 
all of the software components comparing to the respondents from other hospitals in providence. These differences were statistically significant in clinical and supportive scores ( $p$-value $<0.05)$. Furthermore, there was a difference between software design company's scores. Peyvand dadeh and Noskheh pardaz companies received better score in all components (Table 1).

Table 4. Comparing different components of HIS software by hospital types and software design companies

\begin{tabular}{lllllll}
\hline Variables & N & $\begin{array}{l}\text { Overall } \\
\text { score }\end{array}$ & $\begin{array}{l}\text { Clinical } \\
\text { score }\end{array}$ & $\begin{array}{l}\text { Diagnostic } \\
\text { score }\end{array}$ & $\begin{array}{l}\text { Supportive } \\
\text { score }\end{array}$ \\
\hline Hospital teaching & Teaching & 4 & 63.8 & 39.5 & 76.8 & 75.2 \\
status & Non-teaching & 9 & 60.8 & 36.5 & 73.7 & 72.1 \\
& P-value & & 0.437 & 0.351 & 0.534 & 0.534 \\
Hospital ownership & Public & 10 & 59.5 & 33.9 & 73.1 & 71.8 \\
status & Private & 3 & 68.9 & 49.3 & 79.6 & 77.8 \\
& P-value & & 0.307 & 0.126 & 0.307 & 0.126 \\
Geographical location & Capital city & 7 & 66 & 43.7 & 78 & 76.3 \\
& Other cities & 6 & 56.7 & 30.1 & 70.7 & 69.3 \\
& $\begin{array}{l}\text { P-value } \\
\text { Seyvand dadeh }\end{array}$ & 2 & 70.8 & 48.4 & 86.1 & $0.041^{*}$ \\
companies & Tirazheh & 7 & 55.6 & 27.9 & 69.7 & 69.2 \\
& Rahavard & 1 & 64.6 & 46.5 & 76.3 & 76.3 \\
& $\begin{array}{l}\text { Noskheh } \\
\text { pardaz }\end{array}$ & 3 & 68.9 & 49.3 & 77.8 & 77.8 \\
\hline & P-value & & 0.129 & 0.117 & 0.095 & 0.233 \\
\hline
\end{tabular}

* P-value $<0.05$

\section{Discussion}

The highest scores of evaluation relate to laboratory information systems, resource management and registration information systems.

Laboratories have the most amount of consideration because of their vital role in the intensive data management needed in hospitals (Sinard, Castellani, Wilkerson, \& Henricks, 2015). Laboratory systems are automated to enhance their diagnosis, treatment and prevention functions that need the technological and informational infrastructures like HIS (Khajouei, Saghaeiannejad, Jahanbakhsh, \& Mirmohammadi, 2015). This could be the reason for leadership of laboratory systems in Kerman hospitals. Shojaei and his colleagues concluded that laboratory systems have an acceptable status due to their role in command transfers between clinical sectors and testing laboratories, the possibility of priority identification of emergency patients, the ability to view previous results and the ability to mine statistics, according to their 2012 survey evaluating four public hospitals in Kerman (Baghini, Boori Abadi, Joodaki, \& Mollaee, 2015).

The lowest scores in our study were related to telemedicine, staff information and timing systems and decision support system, respectively.

Since telemedicine and decision support system are still underdeveloped in Iran, their low score among the other HIS components are not unexpected. Based on the Jahanbakhsh survey, inadequate IT-security for the protection of e-health-related data, improper training and educational issues, legal challenges, privacy concerns, improper documentation of lessons learned, resistance to the application of new technologies and a lack of recovery plan are the most important barriers to an efficient HIS system in Iran (Baghini, Abadi, Joodaki, \& Mollaee, 2015). Furthermore, according to previous studies, there is a widespread acceptance of HIS in Iranian hospitals, but the problem is in integrating the requirements, needs and infrastructures to the scale of hospital management.

The results of the study showed that all of the software components received better scores in private hospital comparing to the public hospitals. Private hospitals are much more agile and provident than public ones, which could be the reason of their excellence in using different components of their HIS system (Dolan, 2015). Furthermore, medical records are used for different purposes in private and public hospitals, so the financial 
independence of private hospitals is one an important reason for keeping these records (Dehnavieh et al., 2013).

Also all of the software components received better scores in teaching hospital comparing to the non-teaching hospitals. Teaching hospitals are the largest hospitals in the province and are located in the capital of Kerman province, where vendor companies' agents are located too. Therefore, it is not surprising that users in this hospital receive more training related to the HIS. Many studies have emphasized that being more familiar with HIS functions, increase users satisfaction with the software (Ajami \& Mohammadi-Bertiani, 2013; Cho, 2015; Lin, Fan, $\mathrm{Pu}, \mathrm{Li}, \&$ Lian, 2014). Moreover, being the biggest clients of the HIS software vendors, create higher standing for teaching hospitals to receive supportive services (Jafari, Bastani, Ibrahimipour, \& Dehnavieh, 2007).

Hospitals in this study were applying four different HIS software but two of them (i.e. Peyvand dadeh and Noskheh pardaz) were evaluated better than the others in all components. However, their differences were not significant. All privet hospitals use Noskheh pardaz, which has evaluated better than the others. However, while all 10 public hospitals in this study are affiliated to Kerman University of Medical Sciences and financed by the university, they used tree different software. Evidences from this study revealed that Peyvand dadeh has the best practice in public universities in Kerman providence among other HIS software. These findings suggest that all hospitals affiliated to Kerman University of Medical Sciences to use same HIS software and create a consortium to improve regional educational and support programs regarding their HIS.

Furthermore, using integrated HIS facilitates the production of epidemiologic and financial data and create a valuable data bank of hospital services utilization, which helps estimating and planning future hospital services (Whittaker, Mares, \& Rodney, 2013).

It seems that HIS needs more enhancement in Iran's hospitals. Although the status of some indexes is acceptable, there are some indexes that have not entered into the hospitals' infrastructure despite their importance. On the other hand, all of the custodians of the treatment part of the health system need to improve integrally, whether public or private, teaching or non-teaching hospitals, and regardless of their location or software design companies.

\section{Ethical Considerations}

Due to ethical considerations, the presentation letter for Kerman province hospitals from the treatment deputy of the Kerman medical university was received, an agreement with the administrative managers and authority of HIS in hospitals was reached, and they were free to choose whether to fill in the checklists.

\section{Acknowledgements}

The authors really appreciate the hospitals' authorities for participating in joint meetings, and for their collaboration infilling in the checklist, despite of the number of checklists items that required time.

\section{Competing Interests Statement}

The authors declare that there is no conflict of interests regarding the publication of this paper.

\section{References}

Ahmadian, L., Nejad, S. S., \& Khajouei, R. (2015). Evaluation methods used on health information systems (HISs) in Iran and the effects of HISs on Iranian healthcare: A systematic review. International journal of medical informatics, 84(6), 444-453. http://dx.doi.org/10.1016/j.ijmedinf.2015.02.002

Ajami, S., \& Mohammadi-Bertiani, Z. (2013). Training and its impact on hospital information system (HIS) success. Journal of Information Technology \& Software Engin no. 2012.

Baghini, M. S., Abadi, T. N. B., Joodaki, M., \& Mollaee, S. (2015). Progress of hospital information systems in Kerman teaching general hospitals in 2012. Journal of Modern Medical Information Sciences, 1(1), 42-50.

Balaraman, P., \& Kosalram, K. (2013). E-Hospital Management \& Hospital Information Systems-Changing Trends. International Journal of Information Engineering and Electronic Business, 5(1), 50. http://dx.doi.org/10.5815/ijieeb.2013.01.06

Chatzoglou, P. D., Fragidis, L. L., Doumpa, T., \& Aggelidis, P. (2012). Hospital Information System Evaluation. Paper read at 10th International conference on ICT in health.

Cho, V. (2015). A study of the impact of organizational learning on information system effectiveness. International Journal of Business and Information, 2(1).

Dehnavieh, R., Ebrahimipour, H., Molavi-Taleghani, Y., Vafaee-Najar, A., Hekmat, S. N., \& Esmailzdeh, H. (2014). Proactive Risk Assessment of Blood Transfusion Process, in Pediatric Emergency, Using the Health Care Failure Mode and Effects Analysis (HFMEA). Global journal of health science, 7(1), 322. 
http://dx.doi.org/10.5539/gjhs.v7n1p322

Dehnavieh, R., Ebrahimipour, H., Zadeh, M. J., Dianat, M., Hekmat, S. N., \& Mehrolhassani, M. H. (2013). Clinical governance: The challenges of implementation in Iran. International Journal of Hospital Research, 2(1), 1-10.

Dehnavieh, R., Hekmat, S. N., Ghasemi, S., \& Mirshekari, N. (2015). The Vulnerable Aspects of Application of "Health Technology Assessment". International journal of technology assessment in health care, 31(3), 197-198. http://dx.doi.org/10.1017/S0266462315000288

Dehnavieh, R., Rashidian, A., Maleki, M., Tabibi, S. A., Ibrahimi, H. P., \& Hekmat, S. N. (2010). Criteria for Priority-setting in Iran Basic Health Insurance Package: Exploring the Perceptions of Health Insurance Experts. HealthMED, 7(2), 1542.

Dolan, T. (2015). A Six-Dimensional Assessment Tool for e-Government Development Applied to the Homepage Sites of 25 US States. Paper read at Proceedings of the 15th European Conference on eGovernment 2015: ECEG 2015.

Esmailzadeh, H., Mehralhasani, M. H., Dehnavieh, R., Ibrahimipour, H., Hekmat, S. N., \& Sirizi, M. J. (2013). Review of the biotechnology research and development (R and D) countries concerning biological drugs. African Journal of Biotechnology, 10(46), 9459-9466.

Hannan, T. J. (2014). Solving a Health Information Management Problem. An international success story. World hospitals and health services: The official journal of the International Hospital Federation, 51(2), 32-35.

Hekmat, S. N., Dehnavieh, R., Rahimisadegh, R., Kohpeima, V., \& Jahromi, J. K. (2015). Team Attitude Evaluation: An Evaluation in Hospital Committees. Materia Socio-Medica, 27(6), 429. http://dx.doi.org/10.5455/msm.2015.27.429-433

Hekmat, S. N., Tourani, S., Haghdoost, A.-A., Ebrahimipour, H., Mehrolhassani, M. H., \& Dehnavieh, R. (2014). Beneficial and Adverse Effects of the Integration of Medical Education and Health Service in IR. Iran; A Delphi Exercise. Journal of Medical Sciences, 14(1), 21. http://dx.doi.org/10.3923/jms.2014.21.28

Ismail, N. I., Abdullah, N. H., \& Shamsuddin, A. (2015). Adoption of Hospital Information System (HIS) in Malaysian Public Hospitals. Procedia-Social and Behavioral Sciences, 172, 336-343. http://dx.doi.org/10.1016/j.sbspro.2015.01.373

Jafari Sirizi, M., Rashidian, A., Abolhasani, F., Mohammad, K., \& Yazdani, P. Sh. (2008). Qualitative assessment of dimensions and degree of autonomy granting to university hospitals. Hakim Research Journal, 59-71.

Jafari, M., Bastani, P., Ibrahimipour, H., \& Dehnavieh, R. (2007). Attitude of health information system managers and officials of the hospitals regarding the role of information technology in reengineering the business procedures: A qualitative study. Health MED, 6, 208.

Jahanbakhsh, M., Sharifi, M., \& Ayat, M. (2014). The Status of Hospital Information Systems in Iranian Hospitals. Acta Informatica Medica, 22(4), 268. http://dx.doi.org/10.5455/aim.2014.22.268-275

Jouyani, Y., Bahrampour, M., Barouni, M., \& Dehnavieh, R. (2013). Patient Preferences for Hospital Quality: Case Study of Iran. Iranian Red Crescent Medical Journal, 15(9), 804. http://dx.doi.org/10.5812/ircmj. 12851

Khajouei, R., Saghaeiannejad, S., Jahanbakhsh, M., \& Mirmohammadi, M. (2015). Assessment of the Performance of the Laboratory Information System (LIS) Based on the Standards of the American National Standards Institute (ANSI). Journal of Health and Biomedical Informatics, 2(1), 8-16.

Lin, J. J., Fan, M. Q., Pu, H. T., Li, J., \& Lian, J. (2014). An Information Professional Personnel Training Mode Based on Social Requirement of Independent Colleges. Paper read at 2nd International Conference on Teaching and Computational Science. http://dx.doi.org/10.2991/ictcs-14.2014.54

Nurek, M., OlgaKostopoulou, O., Delaney, B. C., \& Esmail, A. (2015). Reducing diagnostic errors in primary care. A systematic meta-review of computerized diagnostic decision support systems by the Linneaus collaboration on patient safety in primary care. European Journal of General Practice, 21(sup1), 8-13.

Sadeghifar, J., Jafari, M., Tofighi, S., Ravaghi, H., \& Maleki, M. R. (2015). Strategic planning, implementation, and evaluation processes in hospital systems: A survey from Iran. Global journal of health science, 7(2), 56.

Schoen, C., Osborn, R., Squires, D., Doty, M., Rasmussen, P., Pierson, R., \& Applebaum, S. (2012). A survey of primary care doctors in ten countries shows progress in use of health information technology, less in other 
areas. Health Affairs, 31(12), 2805-2816. http://dx.doi.org/10.1377/hlthaff.2012.0884

Sinard, J. H., Castellani, W. J., Wilkerson, M. L., \& Henricks, W. H. (2015). Stand-alone laboratory information systems versus laboratory modules incorporated in the electronic health record. Archives of Pathology and Laboratory Medicine, 139(3), 311-318. http://dx.doi.org/10.5858/arpa.2013-0711-SO

Thakare, V., \& Khire, G. (2014). Role of Emerging Technology for Building Smart Hospital Information System. Procedia Economics and Finance, 11, 583-588. http://dx.doi.org/10.1016/S2212-5671(14)00223-8

Turani, S., Haghdoost, A.-A., Maleki, M. R., Ravaghi, H., Tijang, R. D., \& Hekmat, S. N. (2011). The Backgrounds, consequences, and future of the integration of Medical Education and Health Service Delivery System. HealthMed, 5(6), 1847-1856.

Vafaee-Najar, A., Pourtaleb, A., Ebrahimipour, H., \& Dehnavieh, R. (2013). Study of patient's complaints reported over 36 months at a large public educational specialized center of obstetrics and gynecology. Middle-East Journal of Scientific Research, 16(1), 23-29.

Whittaker, M., Mares, R. E., \& Rodney, A. (2013). Mapping health information system competencies for health workers in low-and middle-income countries.

\section{Copyrights}

Copyright for this article is retained by the author(s), with first publication rights granted to the journal.

This is an open-access article distributed under the terms and conditions of the Creative Commons Attribution license (http://creativecommons.org/licenses/by/3.0/). 